\title{
Deregulated microRNAs in triple-negative breast cancer revealed by deep sequencing
}

Yao-Yin Chang ${ }^{1 \dagger}$, Wen-Hung Kuo ${ }^{2 \dagger}$, Jui-Hui Hung ${ }^{1,3}$, Chien-Yueh Lee ${ }^{1,3}$, Yung-Hua Lee ${ }^{3}$, Ya-Chu Chang ${ }^{4}$, Wen-Chun Lin ${ }^{4}$, Cheng-Ying Shen ${ }^{4}$, Chiun-Sheng Huang ${ }^{2}$, Fon-Jou Hsieh ${ }^{5}$, Liang-Chuan Lai ${ }^{6}$, Mong-Hsun Tsai ${ }^{4}$, King-Jen Chang ${ }^{2,7^{*}}$ and Eric Y Chuang ${ }^{1,3^{*}}$

\begin{abstract}
Background: MicroRNAs (miRNAs) are short, non-coding RNA molecules that play critical roles in human malignancy. However, the regulatory characteristics of miRNAs in triple-negative breast cancer, a phenotype of breast cancer that does not express the genes for estrogen receptor, progesterone receptor, and human epidermal growth factor receptor 2 , are still poorly understood.

Methods: In this study, miRNA expression profiles of 24 triple-negative breast cancers and 14 adjacent normal tissues were analyzed using deep sequencing technology. Expression levels of miRNA reads were normalized with the quantile-quantile scaling method. Deregulated miRNAs in triple-negative breast cancer were identified from the sequencing data using the Student's t-test. Quantitative reverse transcription PCR validations were carried out to examine miRNA expression levels. Potential target candidates of a miRNA were predicted using published target prediction algorithms. Luciferase reporter assay experiments were performed to verify a putative miRNA-target relationship. Validated molecular targets of the deregulated miRNAs were retrieved from curated databases and their associations with cancer progression were discussed.
\end{abstract}

Results: A novel 25-miRNA expression signature was found to effectively distinguish triple-negative breast cancers from surrounding normal tissues in a hierarchical clustering analysis. We documented the evidence of seven polycistronic miRNA clusters preferentially harboring deregulated miRNAs in triple-negative breast cancer. Two of these miRNA clusters (miR-143-145 at 5q32 and miR-497-195 at 17p13.1) were markedly down-regulated in triple-negative breast cancer, while the other five miRNA clusters (miR-17-92 at 13q31.3, miR-183-182 at 7q32.2, miR-200-429 at 1p36.33, miR-301b-130b at 22q11.21, and miR-532-502 at Xp11.23) were up-regulated in triplenegative breast cancer. Moreover, miR-130b-5p from the miR-301b-130b cluster was shown to directly repress the cyclin G2 (CCNG2) gene, a crucial cell cycle regulator, in triple-negative breast cancer cells. Luciferase reporter assays showed that miR-130b-5p-mediated repression of CCNG2 was dependent on the sequence of the 3'-untranslated region. The findings described in this study implicate a miR-130b-5p-CCNG2 axis that may be involved in the malignant progression of triple-negative breast cancer.

Conclusions: Our work delivers a clear picture of the global miRNA regulatory characteristics in triple-negative breast cancer and extends the current knowledge of microRNA regulatory network.

Keywords: Triple-negative breast cancer, Deep sequencing, MicroRNA expression, MicroRNA cluster, miR-130b-5p, CCNG2

\footnotetext{
* Correspondence: kingjen@ntu.edu.tw; chuangey@ntu.edu.tw

${ }^{\dagger}$ Equal contributors

2Department of Surgery, College of Medicine, National Taiwan University,

Taipei, Taiwan

${ }^{1}$ Department of Electrical Engineering, Graduate Institute of Biomedical

Electronics and Bioinformatics, National Taiwan University, Taipei, Taiwan

Full list of author information is available at the end of the article
}

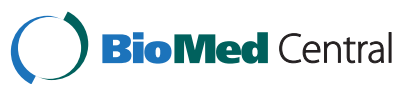

(C) 2015 Chang et al.; licensee Biomed Central. This is an Open Access article distributed under the terms of the Creative Commons Attribution License (http://creativecommons.org/licenses/by/4.0), which permits unrestricted use, distribution, and reproduction in any medium, provided the original work is properly credited. The Creative Commons Public Domain Dedication waiver (http://creativecommons.org/publicdomain/zero/1.0/) applies to the data made available in this article, unless otherwise stated. 


\section{Background}

Breast cancer is a heterogeneous disease that can be classified into several histological forms in current clinical practice. The molecular etiologies among different types of breast cancers are largely different, making the treatment of breast cancer difficult. Triple-negative breast cancer, which is characterized by the lack of expression of the estrogen receptor (ER), the progesterone receptor (PR), and the human epidermal growth factor receptor 2 (HER2), is a type of breast cancer with aggressive tumor behavior. Many targeted treatments, including endocrine therapies and HER2-targeted medicine, are not efficacious for triple-negative breast cancer $[1,2]$. Although previous studies have shown that the vast majority of triple-negative breast cancers display basal-like gene expression features [3,4] the molecular mechanisms driving tumor progression of triple-negative breast cancer still remain unknown.

MicroRNAs (miRNAs) are short ( 21mer) non-coding RNA molecules that are important in gene expression regulation $[5,6]$. The primary role of miRNAs appears to be in the negative regulation of the expression of messenger RNA (mRNA) transcripts. The functional strand of a mature miRNA guides the RNA-induced silencing complex to bind a target mRNA in the 3 '-untranslated region ( 3 '-UTR), initiating translational repression, target mRNA cleavage, or mRNA deadenylation of the target gene. Emerging evidence has shown that aberrant miRNA expression plays a critical role in the tumorigenesis of many human cancers [7-9]. Some miRNAs are shown to possess oncogenic characteristics that promote malignancy of human cancers [10], while some have tumor-suppressing abilities to reduce the production of oncogenic proteins $[11,12]$.

The advent of deep sequencing technology allowed us to explore the largely unknown territory of the miRNA transcriptome in triple-negative breast cancer. Sequencing reads of miRNA expression data from 24 triplenegative breast cancers and 14 adjacent normal tissues were analyzed for the presence of deregulated miRNAs in this study. Differentially expressed miRNAs in triplenegative breast cancer were determined by statistical analyses of the sequencing data and were validated using the quantitative reverse transcription PCR (RT-PCR) method. We identified seven polycistronic miRNA clusters in the human genome harboring 29 deregulated miRNAs in triple-negative breast cancer. Furthermore, our work extends the potential target network of miRNAs by showing that the cyclin G2 gene (CCNG2) is a direct target of miR-130b-5p from the miR-301b-130b cluster. Forced expression of miR-130b-5p was found to significantly repress the endogenous expression levels of CCNG2 in triple-negative breast cancer cells. The findings described in this work may provide insights into the
miRNA regulatory mechanisms underlying the tumorigenicity of triple-negative breast cancer.

\section{Results}

\section{A 25-miRNA signature discriminating triple-negative} breast cancer from adjacent normal tissue

Clinical information on the patients was recorded as shown in Table 1. A total of 113,412,568 miRNA reads were identified from 24 triple-negative breast cancers and 14 adjacent normal tissues after sequence alignment with the human miRNA reference, yielding a median of 2,670,242 miRNA reads per sample from our data (range: 393,305-7,906,634; Additional file 1). Setting a threshold to filter out the miRNAs with extremely low reads (mean expression $<5$ reads across 38 samples), we identified 707 mature miRNAs. The reads from those 707 miRNAs constituted $99.97 \%$ of all miRNA reads in our data for further analysis. The miRNA reads in each sample were normalized using the quantile-quantile scaling method on a $\log _{10}$-scale. Normalized expression data from each of the 707 miRNAs was used as input to generate a PCA plot to visually assess the intrinsic variation in the global miRNA profiles among the samples. The PCA plot showed that the global miRNA portrait was able to roughly separate the majority of triple-negative breast cancers from the adjacent normal tissues (Figure 1A).

Differentially expressed miRNAs between the triplenegative breast cancer group $(\mathrm{N}=24)$ and the adjacent normal tissue group $(\mathrm{N}=14)$ were identified using Student's t-test and the Holm step down procedure for $p$-value adjustment. A list of the top 25 significantly differentially expressed miRNAs (adjusted $p<0.05$ ) was determined as shown in Table 2. Average-linkage hierarchical clustering analysis was performed on the samples using the expression data from the 25 miRNAs (Figure 1B). All triple-negative breast cancers and adjacent normal tissues were distinctively divided into two clusters without any misclassification. To examine whether the 25-miRNA signature contained relevant information in classification of triple-negative breast cancers and normal breast tissues in PCA, each sample was projected onto the principal components using the expression data from the 25 miRNAs (Figure $1 \mathrm{C}$ ). The PCA plot revealed that the triple-negative breast cancers and the adjacent normal tissues were clearly separated into two groups using the 25-miRNA signature.

\section{Polycistronic miRNA clusters harboring differentially expressed miRNAs in triple-negative breast cancer}

The miRNAs residing closely in the same intron, exon, or intergenic region of the human genome were shown to have similar expression patterns in our data. A total of seven miRNA clusters in the human genome were found to be the genomic loci of deregulated miRNAs in 
Table 1 Clinical information on 24 triple-negative breast cancer patients

\begin{tabular}{|c|c|c|c|c|c|c|c|}
\hline Sample ID & Age (years) & Stage & Grade $^{a}$ & Tumor size $^{\mathbf{b}}$ & LYM $^{c}$ & Recurrence status $^{d}$ & Recurrence-free time (years) \\
\hline $452 \_\mathrm{T}$ & 54 & I & 2 & 1 & 0 & 0 & 6.6 \\
\hline 477_T & 27 & I & 3 & 1 & 0 & 0 & 6.5 \\
\hline 593_T & 55 & I & 3 & 1 & 0 & 0 & 5.9 \\
\hline $602 \_T$ & 44 & । & 3 & 1 & 0 & 0 & 5.9 \\
\hline $621 \_T$ & 62 & I & 2 & 1 & 0 & 0 & 5.8 \\
\hline 894_T & 54 & I & 2 & 1 & 0 & 0 & 4.6 \\
\hline $922 \_\mathrm{T}$ & 63 & 1 & 3 & 1 & 0 & 0 & 4.5 \\
\hline 417_T & 33 & $\| \mathrm{A}$ & 3 & 2 & 0 & 0 & 6.8 \\
\hline 507_T & 54 & $\| \mathrm{A}$ & 3 & 1 & 1 & 0 & 6.4 \\
\hline 545_T & 55 & $\| \mathrm{A}$ & 3 & 2 & 0 & 0 & 6.2 \\
\hline 557_T & 60 & $\| \mathrm{A}$ & 3 & 2 & 0 & 0 & 6.1 \\
\hline 574_T & 57 & $\| \mathrm{A}$ & 2 & 2 & 0 & 0 & 6.0 \\
\hline 582_T & 83 & $\| \mathrm{A}$ & 3 & 2 & 0 & 1 & 3.5 \\
\hline 619_T & 37 & $\| \mathrm{A}$ & 3 & 2 & 0 & 1 & 1.8 \\
\hline 673_T & 61 & $\| \mathrm{A}$ & 2 & 2 & 0 & 1 & 0.7 \\
\hline 887_T & 49 & $\| \mathrm{A}$ & 3 & 2 & 0 & 0 & 4.6 \\
\hline 917_T & 51 & $\| \mathrm{A}$ & 3 & 2 & 0 & 0 & 4.5 \\
\hline 918_T & 38 & $\| \mathrm{A}$ & 3 & 2 & 0 & 0 & 4.5 \\
\hline 941_T & 52 & $\| A$ & 3 & 1 & 1 & 0 & 4.4 \\
\hline 677_T & 65 & $\| B$ & 3 & 2 & 1 & 0 & 5.5 \\
\hline 881_T & 58 & $\| B$ & 3 & 2 & 1 & 0 & 4.7 \\
\hline 893_T & 55 & $\| B$ & 3 & 2 & 1 & 1 & 1.5 \\
\hline 291_T & 85 & $\| I A$ & 3 & 3 & 1 & 1 & 1.2 \\
\hline 357_T & 57 & IIIC & 3 & 2 & 1 & 0 & 7.1 \\
\hline
\end{tabular}

${ }^{a}$ Grade: $1 / 2 / 3$, low/intermediate/high.

${ }^{\text {b} T u m o r ~ s i z e: ~} 1 / 2 / 3,<2 \mathrm{~cm} / 2 \mathrm{~cm}-5 \mathrm{~cm} />5 \mathrm{~cm}$.

CLYM, lymph node metastasis: $0 / 1$, negative/positive.

${ }^{\mathrm{d}}$ Recurrence: 0/1, negative/positive.

triple-negative breast cancer (Table 3). Three miRNA members, including miR-143-5p, miR-145-3p, and miR-145-5p, in the miR-143-145 cluster located in the intergenic region at $5 \mathrm{q} 32$ were all significantly downregulated $(p<0.05$; fold change 2.4-3.4). In addition, miR-497-5p and miR-195-5p, in the miR-497-195 cluster located in the intronic region of MIR497HG at 17p13.1, were both significantly down-regulated $(p<0.05$; fold change 3.0-3.3) in triple-negative breast cancer. On the other hand, five other miRNA clusters were found to comprise 24 up-regulated miRNAs in triple-negative breast cancer. These overexpressed miRNAs include four miRNAs $(p<0.05$; fold change 1.9-3.0) in the miR17-92 cluster, five miRNAs $(p<0.05$; fold change 2.5$3.8)$ in the miR-183-182 cluster, four miRNAs $(p<0.05$; fold change 1.7-3.1) in the miR-200b-429 cluster, three miRNAs $(p<0.05$; fold change 2.2-3.6) in the miR-301b$130 \mathrm{~b}$ cluster, and eight miRNAs $(p<0.05$; fold change 2.0-2.6) in the miR-532-502 cluster.

\section{Quantitative RT-PCR validations of the deregulated miRNAs}

To confirm the results obtained from the sequencing data, quantitative RT-PCR validations were carried out to examine the expression levels of the miRNAs residing in the miR-532-502 cluster, the miR-301b-130b cluster, and the miR-497-195 cluster in 19 triple-negative breast cancers and 4 adjacent normal tissues using the comparative $C_{T}$ method [13]. A conceptual diagram that depicts the specific 5p/3p miRNA forms located at each locus in the miR-532-502 cluster is shown in Figure 2A. miR-532-5p (Figure 2B; mean fold change 2.4), miR188-3p (Figure 2C; mean fold change 2.5), miR-362-5p (Figure 2D; mean fold change 4.0), miR-501-3p (Figure 2E; mean fold change 5.3), miR-660-3p (Figure 2F; mean fold change 2.2), and miR-502-5p (Figure 2G; mean fold change 3.0) were all markedly up-regulated in the triplenegative breast cancers compared to the normal breast tissue controls. 


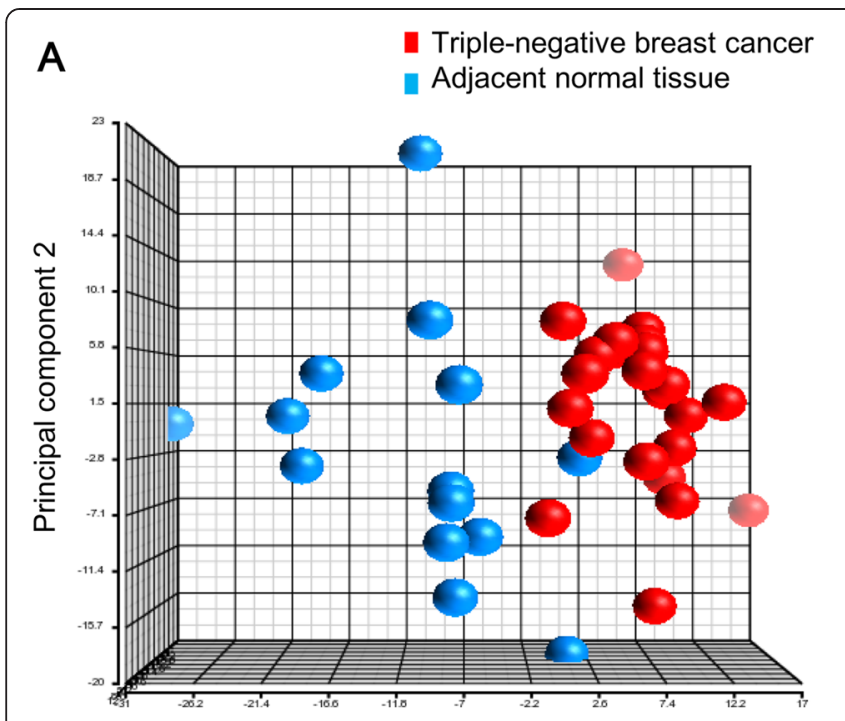

Principal component 1
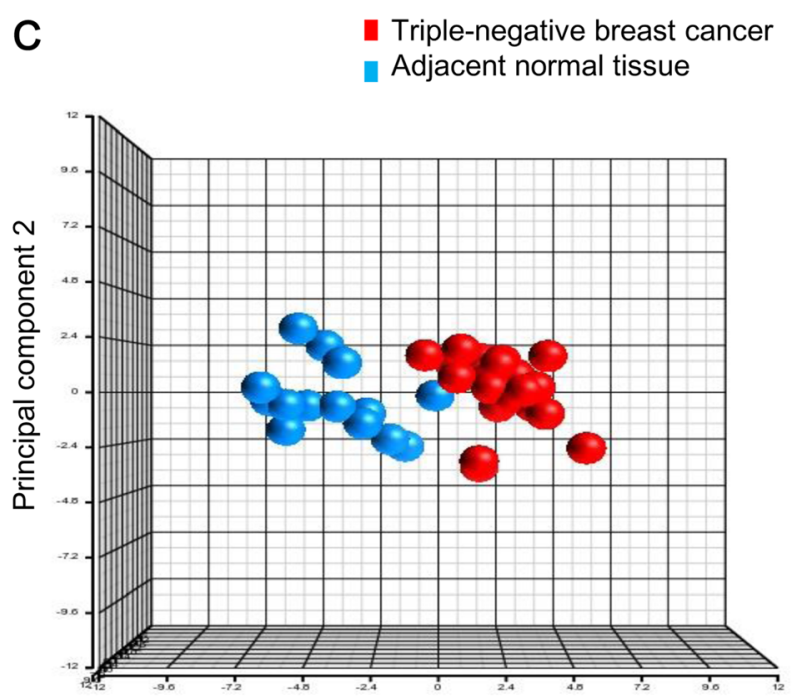

Principal component 1

B

Triple-negative breast cancer

Adjacent normal tissue
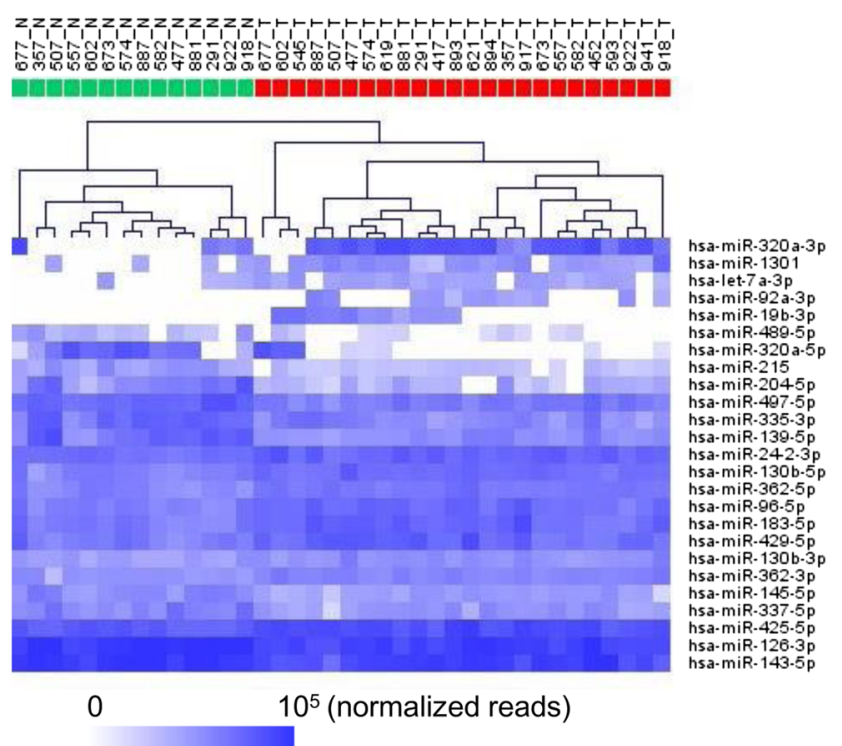

Figure 1 A 25-miRNA expression signature discriminating between triple-negative breast cancers and adjacent normal tissues. (A) The global miRNA expression portrait from each sample was investigated using principal component analysis. Each data point in the principal component plot is composed of gene expression data of 707 mature miRNAs from a sample. Triple-negative breast cancers are shown in red and adjacent normal tissues are shown in blue. (B) Hierarchical clustering analysis of triple-negative breast cancers (red) and adjacent normal tissues (green) was performed using the 25 differentially expressed miRNAs. Each row represents the normalized gene expression data of a miRNA and each column represents a tissue sample. The dendrogram depicts similarities in the gene expression profiles among the samples. (C) Triple-negative breast cancers (red) and adjacent normal tissues (blue) are clearly separated into two groups in the principal component analysis using the gene expression data of the 25 differentially expressed miRNAs.

Figure 3A shows the specific miRNA forms at each locus in the miR-301b-130b cluster. Quantitative RTPCR data confirmed that miR-301b-5p, miR-130b-5p, and miR-130b-3p were all up-regulated in 19 triplenegative breast cancer samples (Figure 3B-D, respectively). Two 5p miRNA forms, miR497-5p and miR-195-5p, located at two different loci in the miR-497-195 cluster are shown in Figure 3E. Compared with the normal breast tissue controls, the expression levels of miR497$5 \mathrm{p}$ were markedly down-regulated (Figure 3F; mean fold change 0.3) in triple-negative breast cancers. Furthermore, miR-195-5p in the same cluster was down-regulated 
Table 2 Twenty-five differentially expressed miRNAs between triple-negative breast cancers and adjacent normal tissues ${ }^{\mathrm{a}}$

\begin{tabular}{|c|c|c|c|c|c|c|c|c|}
\hline \multirow[t]{3}{*}{ miRNA } & \multirow[t]{3}{*}{ Raw $p$ value } & \multirow[t]{3}{*}{ Adjusted $p$ value } & \multicolumn{3}{|c|}{ Triple-negative breast cancer } & \multicolumn{3}{|c|}{ Adjacent normal tissue } \\
\hline & & & \multirow{2}{*}{$\begin{array}{l}\text { Mean } \\
\text { Normalized }\end{array}$} & \multicolumn{2}{|l|}{ Range } & \multirow{2}{*}{$\begin{array}{l}\text { Mean } \\
\text { Normalized }\end{array}$} & \multirow{2}{*}{$\begin{array}{l}\text { Range } \\
\text { Min }\end{array}$} & \multirow[b]{2}{*}{ Max } \\
\hline & & & & Min & Max & & & \\
\hline hsa-miR-215 & $3.21 \mathrm{E}-08$ & $4.66 \mathrm{E}-06$ & 32 & 0 & 86 & 321 & 80 & 928 \\
\hline hsa-miR-497-5p & $2.43 \mathrm{E}-06$ & 3.49E-04 & 1980 & 354 & 6215 & 6520 & 2335 & 18692 \\
\hline hsa-miR-204-5p & 4.69E-06 & $6.71 \mathrm{E}-04$ & 98 & 0 & 569 & 2518 & 37 & 14352 \\
\hline hsa-miR-425-5p & 5.98E-06 & 8.49E-04 & 23266 & 7198 & 57542 & 9180 & 4551 & 16066 \\
\hline hsa-miR-96-5p & $1.34 \mathrm{E}-05$ & $1.89 \mathrm{E}-03$ & 4568 & 898 & 12421 & 1545 & 494 & 3892 \\
\hline hsa-miR-1301 & $1.72 \mathrm{E}-05$ & $2.40 \mathrm{E}-03$ & 592 & 0 & 4777 & 43 & 0 & 188 \\
\hline hsa-miR-335-3p & $2.89 \mathrm{E}-05$ & 4.02E-03 & 1173 & 118 & 4234 & 5591 & 440 & 11145 \\
\hline hsa-miR-183-5p & $5.13 \mathrm{E}-05$ & 7.08E-03 & 6223 & 1305 & 24697 & 1928 & 602 & 5753 \\
\hline hsa-miR-24-2-3p & $6.04 \mathrm{E}-05$ & 8.27E-03 & 6782 & 2062 & 17902 & 3254 & 1553 & 5731 \\
\hline hsa-miR-130b-5p & 7.64E-05 & 1.04E-02 & 3729 & 1413 & 10634 & 1352 & 159 & 2856 \\
\hline hsa-miR-92a-3p & $9.93 \mathrm{E}-05$ & $1.34 \mathrm{E}-02$ & 182 & 0 & 1151 & 0 & 0 & 0 \\
\hline hsa-miR-320a-3p & 1.15E-04 & $1.54 \mathrm{E}-02$ & 11490 & 0 & 29381 & 2273 & 0 & 26275 \\
\hline hsa-miR-130b-3p & 1.23E-04 & $1.64 \mathrm{E}-02$ & 550 & 204 & 1683 & 246 & 110 & 579 \\
\hline hsa-miR-145-5p & $1.47 \mathrm{E}-04$ & 1.94E-02 & 256 & 9 & 603 & 877 & 202 & 2457 \\
\hline hsa-miR-126-3p & $1.55 \mathrm{E}-04$ & 2.03E-02 & 44031 & 16691 & 108893 & 134103 & 29907 & 285350 \\
\hline hsa-miR-139-5p & 1.57E-04 & $2.04 \mathrm{E}-02$ & 693 & 181 & 2537 & 6623 & 307 & 23210 \\
\hline hsa-let-7a-3p & $1.59 \mathrm{E}-04$ & 2.05E-02 & 175 & 0 & 428 & 41 & 0 & 326 \\
\hline hsa-miR-489-5p & 1.59E-04 & 2.05E-02 & 8 & 0 & 63 & 118 & 0 & 483 \\
\hline hsa-miR-362-5p & $1.69 \mathrm{E}-04$ & $2.15 \mathrm{E}-02$ & 2216 & 1186 & 5092 & 1006 & 297 & 2303 \\
\hline hsa-miR-429-5p & $1.91 \mathrm{E}-04$ & $2.40 \mathrm{E}-02$ & 6416 & 1486 & 23455 & 2082 & 406 & 8716 \\
\hline hsa-miR-19b-3p & $2.43 \mathrm{E}-04$ & 3.04E-02 & 569 & 0 & 3214 & 0 & 0 & 0 \\
\hline hsa-miR-362-3p & $2.56 \mathrm{E}-04$ & 3.17E-02 & 1043 & 565 & 2168 & 448 & 43 & 845 \\
\hline hsa-miR-320a-5p & $2.72 \mathrm{E}-04$ & 3.34E-02 & 1354 & 0 & 17859 & 4369 & 0 & 15247 \\
\hline hsa-miR-337-5p & $3.44 \mathrm{E}-04$ & 4.19E-02 & 386 & 9 & 961 & 1309 & 334 & 4086 \\
\hline hsa-miR-143-5p & $3.91 \mathrm{E}-04$ & 4.73E-02 & 43060 & 3104 & 99397 & 102717 & 29650 & 36464 \\
\hline
\end{tabular}

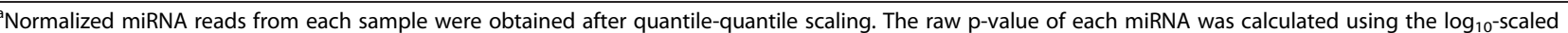
miRNA expression data after quantile-quantile scaling and was adjusted using the Holm step down procedure. Relative expression levels are shown.

(Figure 3G; mean fold change 0.4) in triple-negative breast cancers in the quantitative RT-PCR validations.

\section{miR-130b-5p directly silences endogenous CCNG2}

\section{expression levels in MDA-MB-231 cells}

To understand how these deregulated miRNAs might function in triple-negative breast cancer, we performed target prediction of the miRNAs as described in the Methods section. Among those putative target candidates, five potential tumor suppressors, including CCNG2, FOXP1, NRG1, LRIG1, and TNFSF10, were shown to have a direct binding site with miR-130b-5p. We were particularly interested in CCNG2, because it was a crucial regulatory gene in cell cycle control $[14,15]$. Previous studies have functionally linked the decrease or complete loss of CCNG2 in human cancer to uncontrolled cell proliferation $[16,17]$. We thus further characterized the regulatory mechanism of miR-130b-5p in relation to CCNG2 in cells.
A schematic diagram of the putative miR-130b-5p binding site in the 3 '-UTR of CCNG2 is shown in Figure 4A. Two luciferase reporter constructs, Luc-CCNG2-UTR and LucCCNG2-UTR-mt3, were made to test whether miR-130b$5 \mathrm{p}$ might directly target CCNG2 in the putative binding site. The possible binding sequences in the $3^{\prime}$-UTR of CCNG2 were carried by the CCNG2 gene reporter, LucCCNG2-UTR. The other luciferase reporter construct, Luc-CCNG2-UTR-mt3, was made to carry three mutated nucleotides at the putative binding site to disrupt binding of miR-130b-5p to CCNG2. The luciferase reporter assays revealed that miR-130b-5p significantly reduced the luciferase activity of the wild-type CCNG2 gene reporter, but not that of the site-directed mutant (Figure 4B), suggesting that miR-130b-5p had a suppressive effect at the predicted binding site in the 3 ' -UTR of CCNG2.

Next, we examined whether miR-130b-5p might repress endogenous CCNG2 expression in triple-negative breast 
Table 3 Polycistronic miRNA clusters harboring deregulated miRNAs between triple-negative breast cancers and adjacent normal tissues $^{\mathrm{a}}$

\begin{tabular}{|c|c|c|c|c|c|c|c|c|}
\hline \multirow[t]{3}{*}{ miRNA } & \multirow[t]{3}{*}{$p$ value } & \multicolumn{3}{|c|}{ Triple-negative breast cancer } & \multicolumn{3}{|c|}{ Adjacent normal tissue } & \multirow[t]{3}{*}{ Fold change ${ }^{b}$ in expression } \\
\hline & & \multicolumn{3}{|c|}{$\begin{array}{ll}\text { Mean } & \text { Range }\end{array}$} & \multirow{2}{*}{$\begin{array}{l}\text { Mean } \\
\text { Normalized }\end{array}$} & \multicolumn{2}{|c|}{ Range } & \\
\hline & & Normalized & Min & Max & & Min & Max & \\
\hline
\end{tabular}

Down-regulated miRNAs in triple-negative breast cancer

miR-143-145 cluster (Chromosome 5 q32, intergenic)

$\begin{array}{lllllllll}\text { hsa-miR-143-5p } & 3.91 \mathrm{E}-04 & 43060 & 3104 & 99397 & 102717 & 29650 & 364644 & 2.4 \\ \text { hsa-miR-145-3p } & 7.36 \mathrm{E}-03 & 128335 & 8310 & 287564 & 404278 & 43410 & 1212945 & 3.2 \\ \text { hsa-miR-145-5p } & 1.47 \mathrm{E}-04 & 256 & 9 & 603 & 877 & 202 & 2457 & 3.4 \\ \text { miR-497-195 cluster } & \text { (Chromosome } & \mathbf{1 7} \text { p13.1, MIR497HG intron 1) } & & & & \\ \text { hsa-miR-497-5p } & 2.43 \mathrm{E}-06 & 1980 & 354 & 6215 & 6520 & 2335 & 18692 & 3.3 \\ \text { hsa-miR-195-5p } & 3.18 \mathrm{E}-03 & 239 & 52 & 504 & 721 & 128 & 2492 & 3.0\end{array}$

Up-regulated miRNAs in triple-negative breast cancer

miR-17-92 cluster (Chromosome 13 q31.3, C13orf25 intron 3)

$\begin{array}{lllllllll}\text { has-miR-17-3p } & 2.66 \mathrm{E}-02 & 40467 & 7676 & 137962 & 21080 & 5047 & 67282 & 1.9 \\ \text { has-miR-18a-3p } & 1.43 \mathrm{E}-02 & 6362 & 904 & 26300 & 2141 & 0 & 11488 & 3.0 \\ \text { has-miR-19b-3p } & 9.93 \mathrm{E}-05 & 569 & 0 & 3214 & 0 & 0 & 0 & \text { N/A } \\ \text { has-miR-92a-3p } & 2.43 \mathrm{E}-04 & 182 & 0 & 1151 & 0 & 0 & 0 & \text { N/A }\end{array}$

miR-183-182 cluster (Chromosome 7 q32.2, intergenic)

$\begin{array}{lllllllll}\text { hsa-miR-183-3p } & 5.42 \mathrm{E}-03 & 160 & 29 & 431 & 63 & 0 & 155 & 2.5 \\ \text { hsa-miR-183-5p } & 5.13 \mathrm{E}-05 & 6223 & 1305 & 24697 & 1928 & 602 & 5753 & 3.2 \\ \text { hsa-miR-96-3p } & 2.70 \mathrm{E}-03 & 65 & 0 & 163 & 26 & 0 & 106 & 2.5 \\ \text { hsa-miR-96-5p } & 1.34 \mathrm{E}-05 & 4568 & 898 & 12421 & 1545 & 494 & 3892 & 3.0 \\ \text { hsa-miR-182-5p } & 4.76 \mathrm{E}-03 & 13189 & 2441 & 40871 & 3501 & 7 & 7623 & 3.8 \\ \text { miR-200b-429 cluster (Chromosome 1 p36.33, intergenic) } & & & & & \\ \text { hsa-miR-200b-3p } & 2.65 \mathrm{E}-02 & 694 & 156 & 2348 & 398 & 35 & 1061 & 1.7 \\ \text { hsa-miR-200a-3p } & 2.46 \mathrm{E}-02 & 77 & 0 & 362 & 40 & 0 & 296 & 1.9 \\ \text { hsa-miR-200a-5p } & 1.25 \mathrm{E}-03 & 46665 & 1800 & 160398 & 15886 & 2412 & 46254 & 2.9 \\ \text { hsa-miR-429-5p } & 1.91 \mathrm{E}-04 & 6416 & 1486 & 23455 & 2082 & 406 & 8716 & 3.1\end{array}$

miR-301b-130b cluster (Chromosome 22 q11.21, PPIL2 exon 2)

$\begin{array}{lllllllll}\text { hsa-miR-301b-5p } & 1.41 \mathrm{E}-03 & 323 & 103 & 1483 & 89 & 0 & 244 & 3.6 \\ \text { hsa-miR-130b-3p } & 1.23 \mathrm{E}-04 & 550 & 204 & 1683 & 246 & 110 & 579 & 2.2 \\ \text { hsa-miR-130b-5p } & 7.64 \mathrm{E}-05 & 3729 & 1413 & 10634 & 1352 & 159 & 2856 & 2.8\end{array}$

miR-532-502 cluster (Chromosome X p11.23, CLCN5 intron 3)

$\begin{array}{lllllllll}\text { hsa-miR-532-5p } & 1.58 \mathrm{E}-02 & 514 & 125 & 2731 & 253 & 31 & 990 & 2.0 \\ \text { hsa-miR-188-3p } & 2.72 \mathrm{E}-02 & 1159 & 509 & 5061 & 520 & 0 & 1258 & 2.2 \\ \text { hsa-miR-362-3p } & 2.56 \mathrm{E}-04 & 1043 & 565 & 2168 & 448 & 43 & 845 & 2.3 \\ \text { hsa-miR-362-5p } & 1.69 \mathrm{E}-04 & 2216 & 1186 & 5092 & 1006 & 297 & 2303 & 2.2 \\ \text { hsa-miR-501-3p } & 2.13 \mathrm{E}-02 & 335 & 125 & 1439 & 143 & 0 & 258 & 2.3 \\ \text { hsa-miR-660-3p } & 3.00 \mathrm{E}-02 & 11371 & 5546 & 23930 & 4438 & 0 & 9196 & 2.6 \\ \text { hsa-miR-502-3p } & 1.01 \mathrm{E}-02 & 144 & 60 & 339 & 68 & 0 & 129 & 2.1 \\ \text { hsa-miR-502-5p } & 2.55 \mathrm{E}-02 & 354 & 98 & 717 & 174 & 0 & 351 & 2.0\end{array}$

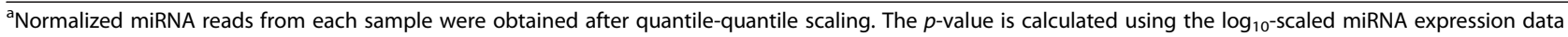
without the Holm adjustment. Relative expression levels are shown.

${ }^{\mathrm{b}}$ Fold change was obtained by comparing the mean normalized miRNA expression reads between the triple-negative breast cancers and adjacent normal tissues. 


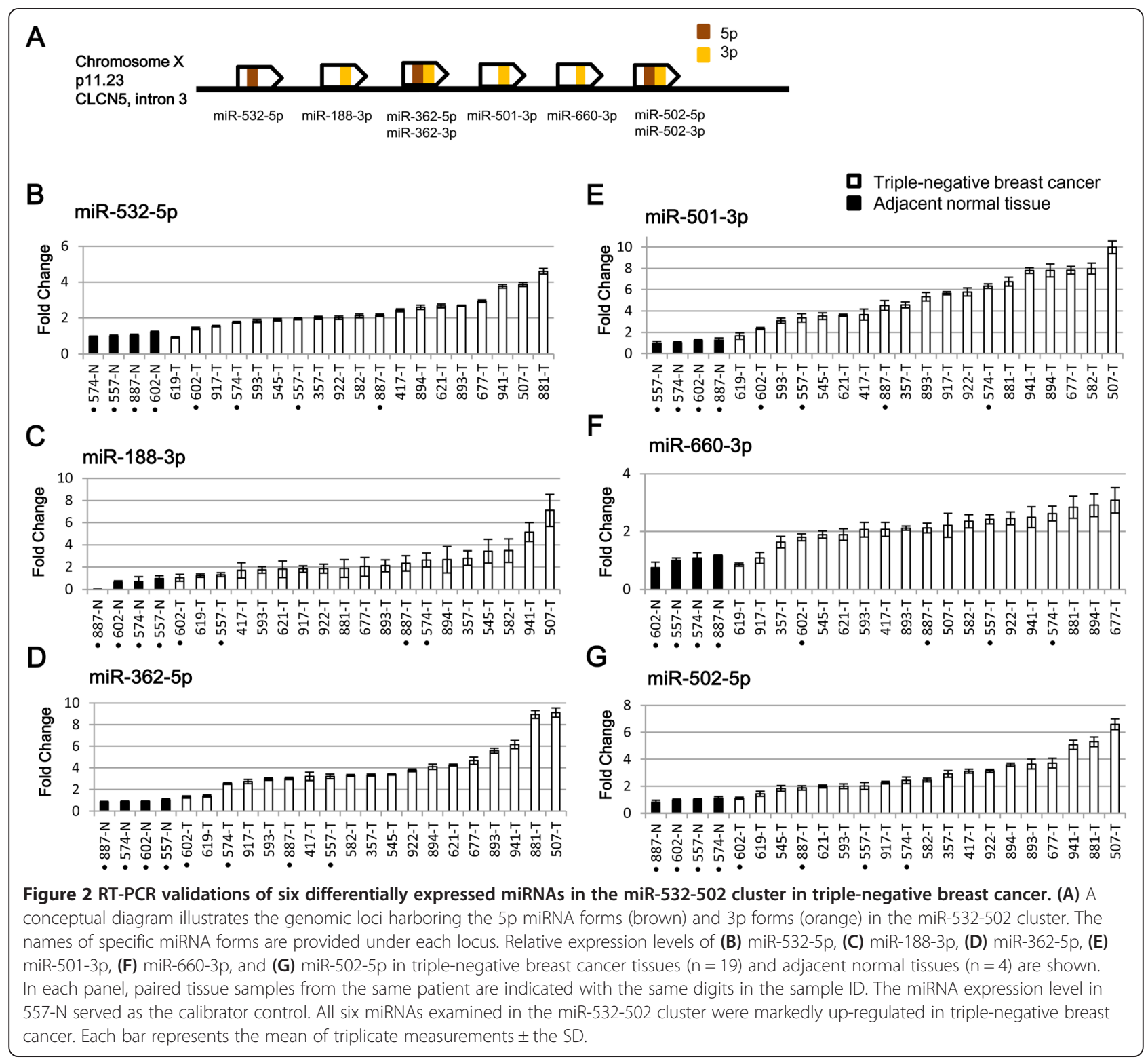

cancer cells. Overexpression of miR-130b-5p was carried out in MDA-MB-231 cells using plasmid DNA transfection of a lentiviral vector containing the precursor sequence of miR-130b-5p. Quantitative RT-PCR data confirmed that the endogenous expression levels of CCNG2 were significantly repressed in MDA-MB-231 cells after overexpression of miR-130b-5p (Figure 4C). Compared with negative controls, a marked reduction $(>50 \%)$ in CCNG2 expression level was observed in the miR-130b5p-overexpressed MDA-MB-231cells.

The functional relevance of miR-130b-5p in cell cycle regulation with triple-negative breast cancer cells was analyzed in this study. After overexpression of miR130b-5p, MDA-MB-231 cells showed significant alterations $(p<0.05)$ in cell cycle profile (Additional file 2). A significant decrease in G1 phase and increases in S and $\mathrm{G} 2 / \mathrm{M}$ phases were observed with the miR-130b-5p-overexpressed MDA-MB-231cells in the cell cycle analysis. These results strongly support that miR-130b-5p plays a role in cell cycle progression through suppression of CCNG2 expression in triple-negative breast cancer.

We then investigated an association between miR130b-5p expression and malignancy of triple-negative breast cancer from the sequencing data in our cohort. Sequencing data of miR-130b-5p expression were shown to be significantly associated with tumor progression in both early-stage triple-negative breast cancers $(p=0.002$; $\mathrm{n}=7)$ and advanced stage triple-negative breast cancers $(p<0.001 ; \mathrm{n}=17)$ in Figure 4D. The expression levels of CCNG2 in our cohort of 38 samples were also measured 


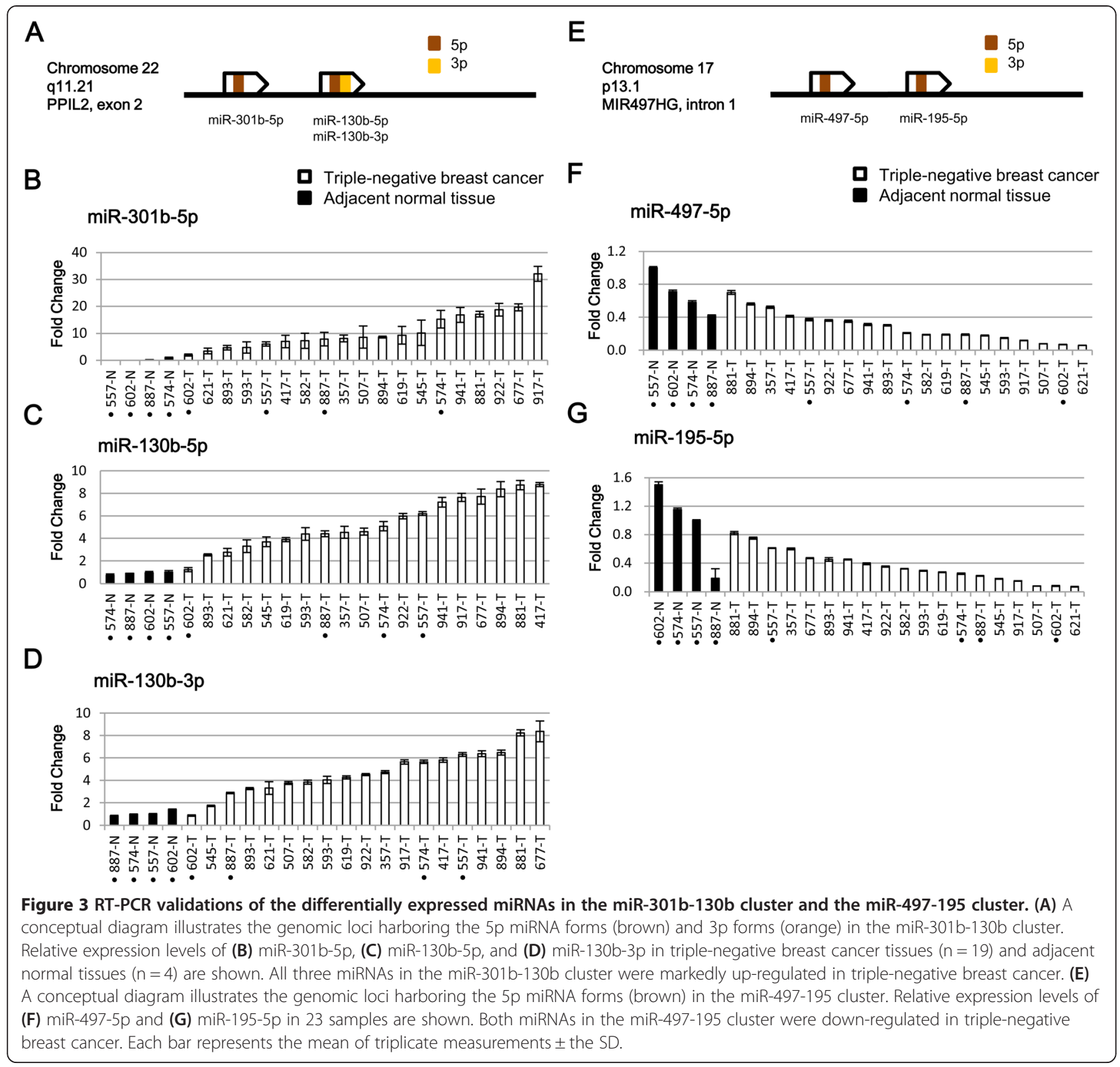

using Agilent microarray (Human 1A v2). A direct correlation between high miR-130b-5p expression $(p<0.001$; fold change 2.8) and low CCNG2 expression $(p<0.001$; fold change 0.5 ) was observed in our triple-negative breast cancer samples as compared with normal breast tissue controls (Additional file $3 \mathrm{~A}$ and $\mathrm{B}$ ).

\section{Discussion}

Sequencing data of miRNA expression reads from 24 triple-negative breast cancers and 14 adjacent normal tissues were analyzed in this study. Deregulated miRNAs were identified from the statistical analyses and a panel of the top 25 deregulated miRNAs was found to be an effective discriminator between triple-negative breast cancers and adjacent normal tissues. Deep sequencing technology allowed us to generate a comprehensive insight into the cellular transcriptome in triple-negative breast cancer that led to the identification of many more deregulated miRNAs not described in previous studies $[18,19]$. For example, aberrant expression from the miR532-502 cluster in triple-negative breast cancer was first documented in this study. Interestingly, the expression level of the tumor suppressor gene RUNX3 was found to be inversely correlated with that of miR-532-5p from the miR-532-502 cluster in primary melanomas [20].

A number of the validated target genes of the deregulated miRNAs in our findings were shown to be involved in human cancer signaling cascades (Additional file 4). 


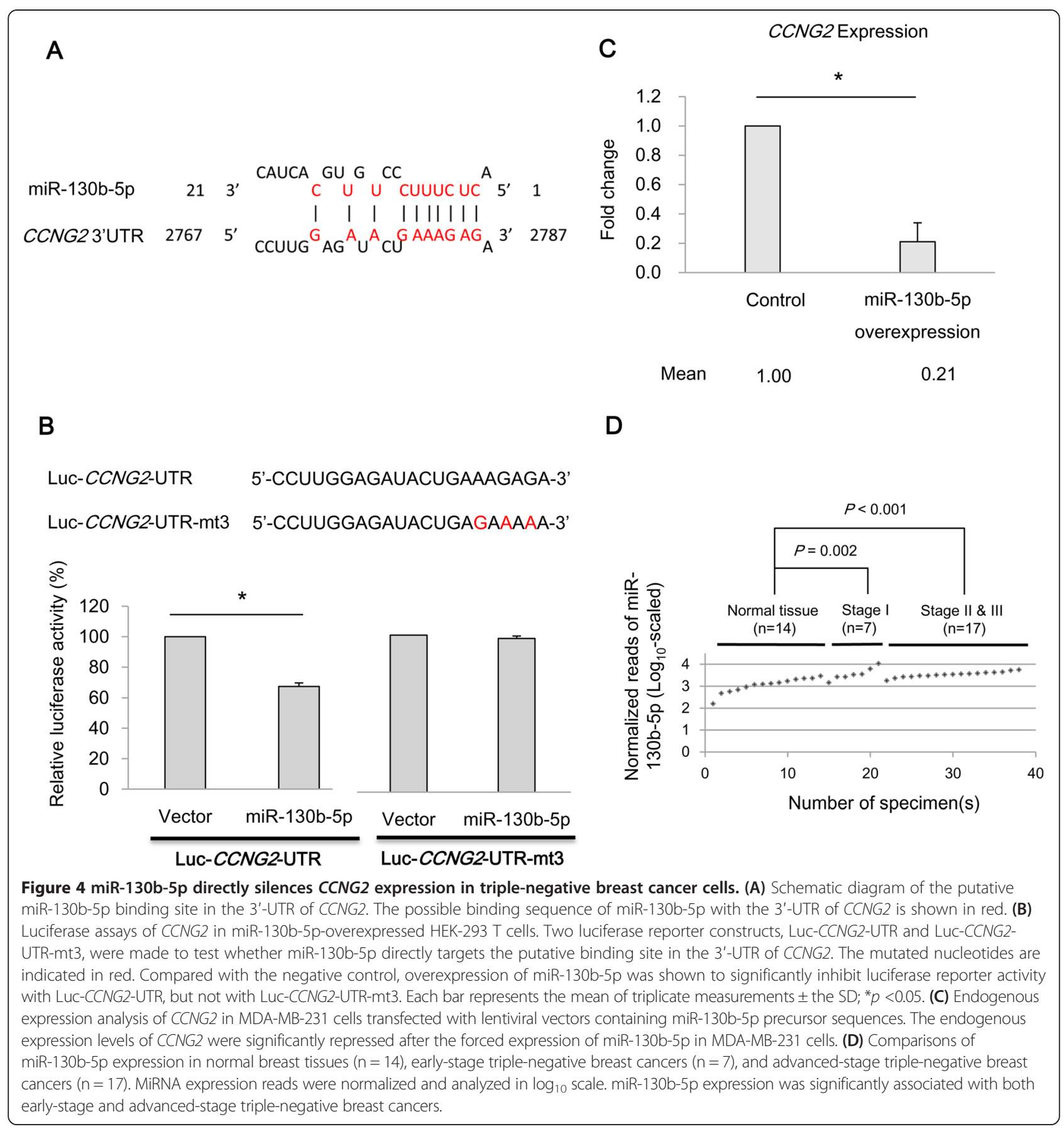

For example, we observed that both miR-143-5p and miR145-5p in the miR-143-145 cluster were down-regulated in triple-negative breast cancer. Down-regulation of these two miRNAs was previously described in lung cancer, colon cancer, and bladder cancer [21-23]. The protooncogene c-Myc was a direct target for miR-145-5p, and introduction of miR-145-5p repressed c-Myc expression and tumor growth both in vitro and in vivo [11]. The insulin receptor substrate-1 (IRS-1), previously known as a major docking protein for both the type 1 insulin- like growth factor receptor and the insulin receptor in cancer cell growth and proliferation signaling, was also a validated target of miR-145-5p [24]. In addition, Sachdeva et al. reported that cell invasion ability was significantly inhibited by miR-145-5p, in part due to the silencing of the metastasis gene MUC1 [25].

Deregulation of the miR-497-195 cluster has been previously addressed in breast cancer. Li et al. described that miR-497-5p and miR-195-5p in this cluster were both down-regulated in human breast cancer tissues and 
cell lines [26]. However, the study did not specifically determine whether the deregulation of these two miRNAs observed in breast cancer cell lines was also observed in triple-negative breast cancer tissues. Our results provide evidence showing in vivo that both miR-497-5p and miR195-5p are down-regulated in triple-negative breast cancer tissues. Furthermore, the methylation state of $\mathrm{CpG}$ islands in the promoter region upstream of the miR497-195 cluster was responsible for the down-regulation of those two miRNAs in breast cancer, and direct targets of miR-1955p included CCND1 and RAF1 [26]. Moreover, introduction of miR-195-5p was shown to inhibit cancer cell colony formation in vitro [26] and tumor development in nude mice [27], suggesting that ectopic expression of miR-195-5p may be involved in the tumorigenesis of breast cancer.

We were able to identify CCNG2 as a direct target of miR-130b-5p in triple-negative breast cancer. Luciferase reporter assays revealed that miR-130b-5p-mediated repression of CCNG2 is dependent on the sequence of the 3 '-UTR. CCNG2 is known as a negative regulator of cell cycle progression. Previous microarray analyses have shown that elevated CCNG2 expression induces cell cycle arrest during responses to various types of growth inhibitory effects, such as hypoxia, oxidative stress, and heat shock $[17,28,29]$. The CCNG2 protein directly interacts with the catalytic subunit of protein phosphatase $2 \mathrm{~A}$ to form active complexes that inhibit cell cycle progression [15]. Increasingly, the evidence suggests that CCNG2 is crucially involved in human cancer signaling pathways [30-32]. For example, CCNG2 was a primary target gene of estrogen-occupied estrogen receptor and that its expression was rapidly down-regulated by estrogens in MCF-7 breast cancer cells [31]. Moreover, CCNG2 promoter activity was found to be regulated by Nodal signaling in ovarian cancer cells and silencing of CCNG2 expression significantly increased cell proliferation [32].

Gene expression levels of CCNG2 between triplenegative breast cancers and normal breast tissues were further investigated using a published microarray dataset [GEO:GSE53752]. The gene expression levels of CCNG2 in triple-negative breast cancers $(\mathrm{n}=51)$ were significantly lower $(p<0.001$; fold change 1.9) than those in normal breast tissues $(\mathrm{n}=25)$ in the microarray data (Additional file 5). Of added interest, recently CCNG2 was found to be an important prognostic factor for triple-negative breast cancer patients. In an analysis of a cohort of 250 primary triple-negative breast cancer samples from eight clinically annotated gene expression datasets, triplenegative breast cancer patients with low expression levels of SHARP1 and CCNG2 had a significantly higher probability of developing metastases and of reduced survival [33]. It is thus important that we identified CCNG2 as a direct target of miR-130b-5p. The ability of miR-130b-5p to repress CCNG2 expression may enhance malignancy by accelerating cell cycle transition in triple-negative tumor cells.

\section{Conclusions}

Our work clearly depicts the global miRNA regulatory characteristics in triple-negative breast cancer. The 25miRNA signature determined in this study may be used as a functional tool to distinguish triple-negative breast cancer tissues from normal breast tissues. Seven polycistronic miRNA clusters preferentially harboring deregulated miRNAs were identified in triple-negative breast cancer. Moreover, we extended the current knowledge of microRNA regulatory network by showing that miR130b-5p in the miR-301b-130b cluster directly silences CCNG2 in triple-negative breast cancer. It would be interesting to test whether this novel regulatory mechanism of miR-130b-5p and its CCNG2 target in triple-negative breast cancer may be involved in other malignancies as well. Our findings not only provide insight into the miRNA regulatory mechanisms in triple negative breast cancer, but also shed light on the identification of potential therapeutic targets for this disease.

\section{Methods}

\section{Breast cancer and normal tissue samples}

Twenty-four triple-negative breast cancer samples and 14 adjacent normal tissue samples were collected from breast cancer patients during surgeries at National Taiwan University Hospital (NTUH, Taipei, Taiwan). All triplenegative breast cancer samples were invasive ductal carcinomas and were negative in immunohistochemical analyses of ER, PR, and HER2. AJCC/UICC TNM staging system was used for tumor classification. Treatment of each patient followed the National Comprehensive Cancer Network (http://www.nccn.org/) guidelines. All samples were neoadjuvant-free and were collected before systemic chemotherapy treatments. Written informed consent was obtained from each patient who participated in this study. All human tissues used in this study were approved by the institutional review board at NTUH.

\section{Small RNA library preparation}

Total RNA was extracted from each sample for the preparation of a small RNA library. The small RNA library was constructed from total RNA using the SOLiD Total RNA-Seq Kit (Applied Biosystems, Foster City, CA, USA). Integrity of each small RNA library was examined using an RNA 6000 Nano Chip (Agilent, Santa Clara, CA, USA), a Small RNA Chip (Agilent), and the Bioanalyzer (Agilent) according to the manufacturer's instructions. 


\section{Deep sequencing experiments}

Upon completion of PCR amplification, the small RNA libraries were purified using the SOLiD Library Micro Column Purification Kit (Applied Biosystems) and hybridized to the template beads using the SOLiD EZ bead system (Applied Biosystems). The template beads were amplified and deposited onto a tray for small RNA ligation sequencing by the SOLiD 4 System (Applied Biosystems). The sequencing data were uploaded to the Gene Expression Omnibus (GEO) with an accession number of GSE40049.

\section{Sequence alignment of miRNA reads}

All reads obtained from ligation sequencing were first screened to filter out the reads containing ribosomal RNA, transfer RNA, and adaptor sequences. The remaining reads were then aligned to the human miRNA reference (miRBase v17.0) and the human genome reference (RefSeq Hg19) using the Small RNA Analysis Tool (Applied Biosystems). In the sequence alignment, only one mismatch was allowed for the first 16 bases of a miRNA read. The maximum number of permitted mismatches of a miRNA read was set at 4 . Those reads that were not uniquely mapped to the miRBase reference were disregarded to eliminate ambiguous alignments.

\section{Statistical analyses}

The quantile-quantile scaling method [34] was performed for the normalization of miRNA expression reads in $\log _{10^{-}}$ scale. All miRNA expression reads in each dataset were linearly scaled to fit into a miRNA expression reference made of the mean expression value of each miRNA from 38 samples. Principal component analysis (PCA) was performed to analyze the miRNA expression profiles between triple-negative breast cancers and adjacent normal tissues with the Partek Genomics Suite (Partek Incorporated, St. Louis, MO, USA). Significant differences in expression of miRNAs from the triple-negative breast cancers and adjacent normal tissues were identified using two-tailed Student's t-tests. The Holm step down procedure was used to counteract multiple comparisons. A $p$-value of $<0.05$ was considered significant. The miRNAs with a fold change $>2$ and mean expression difference $>100$ reads between the two groups were investigated in the hierarchical clustering analysis using Genesis software (version 1.7.5).

\section{Quantitative RT-PCR validation of miRNA expression}

Differentially expressed miRNAs identified from our sequencing data were validated using quantitative RT-PCR in 19 triple-negative breast cancer samples and 4 adjacent normal tissue samples. Total RNA was extracted from each sample and then reverse transcribed into miRNAspecific cDNA following the standard protocol of the TaqMan MicroRNA Reverse Transcription Kit (Applied
Biosystems). Relative quantification of miRNA expression in each sample was obtained using the comparative threshold $\left(\mathrm{C}_{\mathrm{T}}\right)$ method [13]. Expression of U6 small nuclear RNA was used as the endogenous control.

\section{Putative target prediction}

Potential mRNA target genes of a miRNA were searched using the miRanda [35] and Diana [36] target prediction algorithms. Putative target candidates having complementary base-pairing matches in the 3'-UTR for the indicated miRNA seed region were obtained. Biological functions associated with the target genes were investigated using Ingenuity Pathway Analysis software (Ingenuity Systems, Redwood City, CA, USA).

\section{Experimentally validated miRNA target genes}

Experimentally validated miRNA target genes were retrieved from TarBase [37] and miRecords [38] using the miRSystem search engine [39]. The molecular pathways encompassing the validated target genes were investigated using Ingenuity Pathway Analysis software.

\section{Cell lines}

MDA-MB-231 and HEK-293 T were obtained from Bioresource Collection and Research Center (Taiwan). The cell lines were tested and authenticated by Genelabs Life Science (Taiwan) using STR-PCR profiling.

\section{Vectors}

miR-130b-5p was cloned into a lentiviral vector PreMiR130b (System Biosciences, Mountain View, CA, USA) that was used to overexpress the miRNA in MDA-MB231 cells. Expression of miR-130b-5p was verified and quantified using KAPA PROBE Fast qPCR Master Mix (Kapa Biosystems, Boston, MA, USA), and the LightCycler 480 System (Roche, Basel, Switzerland).

A CCNG2 luciferase reporter construct was made by introducing the CCNG2 3'-UTR carrying a predicted miR-130b-5p binding site (5'-CCTTGGAGATACTGA AAGAGA-3') into the pmirGLO control vector (Promega, Madison, WI, USA). Site-directed mutagenesis of the putative miR-130b-5p binding site was made using a facile PCR procedure [40]. All PCR products were verified by DNA sequencing before use.

\section{Luciferase assay}

Luciferase assays were performed with HEK-293 T cells using the Dual-Glo ${ }^{\circ}$ Luciferase Assay System (Promega). Cells were transfected with PreMiR-130b lentiviral vectors using TransIT ${ }^{-}$-2020 transfection reagent (Mirus Bio, Madison, WI, USA). Forty-eight hours after transfection, the cells were then harvested and lysed for the luciferase assay. Renilla luciferase signals were used for normalization according to the manufacturer's protocol. 


\section{Cell cycle analysis}

$3 \times 10^{4}$ cells of MDA-MB-231 cells were seeded in a 24well plate. miR-130b-5p or empty vector (control) were overexpressed in the MDA-MB-231 cells. Cell synchronization was performed using double thymidine block. $2 \mathrm{mM}$ of thymidine was added into cells and cells were incubated at $37^{\circ} \mathrm{C}$ for 16 hours. To remove thymidine, cells were washed with PBS and incubated with fresh media at $37^{\circ} \mathrm{C}$ for 8 hours. $2 \mathrm{mM}$ of thymidine was added into cells and cells were incubated at $37^{\circ} \mathrm{C}$ for 16 hours. To release the cells from thymidine block, cells were washed with PBS and incubated with fresh media and collected after 14 hours. The cells were harvested by trypsinization and washed twice with cold PBS. The cells were fixed by $0.5 \mathrm{~mL}$ of cold $95 \%$ ethanol and were kept at $-20^{\circ} \mathrm{C}$ overnight. The ethanol was removed and cells were washed twice with PBS. The cells were resuspended in $500 \mu \mathrm{L}$ of PI solution $(10 \mu \mathrm{g} / \mathrm{mL}$ of propidium iodide, $0.2 \mathrm{mg} / \mathrm{mL}$ of RNaseA and $0.1 \%$ Triton X-100). Cell cycle profile was analyzed by fluorescence-activated cell sorter (FACS) analysis.

\section{Gene expression microarray experiments}

Gene expression data of CCNG2 in our cohort of 38 samples (14 normal breast tissues and 24 triple-negative breast cancer samples) were analyzed using Agilent Human 1A (version 2) microarray platform. Microarray experiments were performed following the manufacturer's instructions. Microarray data were normalized using Quantile normalization before statistical analyses.

\section{Online supporting information}

The sequencing data were uploaded to the Gene Expression Omnibus (GEO) with an accession number of GSE40049.

\section{Additional files}

Additional file 1: Raw miRNA reads from triple-negative breast cancers $(\mathrm{N}=24)$ and adjacent normal tissues $(\mathrm{N}=14)$ obtained using the high-throughput sequencer.

Additional file 2: Cell cycle analysis showed significant alterations in cell cycle profile in MDA_MB-231 cells after miR-130b-5p overexpression. MDA-MB-231 cells were transfected with empty vectors (control) or vectors containing miR-130b-5p precursor sequence. Compared with vector controls, there was a significant decrease with the cell population in G1 phase along with increases in S and G2/M phases in the miR-130b-5p-overexpressed MDA_MB-231 cells. The data were analyzed using paired t-test. ${ }^{*} p<0.05$. Bars, \pm SD

Additional file 3: Expression levels of miR-130b-5p and CCNG2 were inversely regulated between normal breast tissues $(n=14)$ and triple-negative breast cancers $(n=24)$ in our cohort of 38 samples. (A) Normalized deep-sequencing reads of miR-130b-5p in triple-negative breast cancers and normal breast tissue controls are shown in the box plots. miR-130b-5p expression was significantly ( $p<0.001)$ up-regulated in triple-negative breast cancers. The $p$ value was calculated with the normalized $\log _{10}$-scaled miRNA reads using the parametric t-test. (B) Agilent microarray data of CCNG2 expression in triple-negative breast cancers and normal breast tissue controls in our cohort are shown in the box plots. Microarray data were normalized using quantile normalization before the statistical analyses. CCNG2 was significantly $(p<0.001)$ down-regulated in triple-negative breast cancers. Fold change was calculated using the mean expression data from the two groups. The $p$ value was obtained using the parametric t-test.

Additional file 4: Experimentally validated miRNA-target gene relationships of the deregulated miRNAs in triple-negative breast cancer. The associations between miRNAs and human cancer were retrieved from curated databases.

Additional file 5: Comparison of CCNG2 expression levels between normal breast tissues $(n=25)$ and triple-negative breast cancers $(n=51)$ from a published microarray dataset [GEO:GSE33926]. The microarray data were obtained using the Agilent Human 1 A (version 2) platform. Quantile normalization was performed on the microarray data before the parametric t-test analysis. The $p$-value was adjusted using Bonferroni correction for multiple comparisons. The microarray data revealed that, compared with normal breast tissues, CCNG2 was significantly down-regulated in triple-negative breast cancers.

\section{Abbreviations}

miRNA: MicroRNA; CCNG2: Cyclin G2; ER: Estrogen receptor; PR: Progesterone receptor; HER2: Human epidermal growth factor receptor 2;

mRNA: messenger RNA; UTR: Untranslated region; RT-PCR: Reverse transcription PCR; NTUH: National Taiwan University Hospital;

IHC: Immunohistochemical; GEO: Gene Expression Omnibus; PCA: Principal component analysis; SD: Standard deviation.

\section{Competing interests}

The authors declare that they have no competing interests.

\section{Authors' contributions}

YYC and WHK carried out the miRNA transcriptome study, performed bioinformatics analyses, and drafted the manuscript. JHH and CYL participated in the miRNA sequencing alignment. YHL, WCL, and CYS performed RT-PCR validation and luciferase assay experiments. CSH, FJH, LCL, and MHT participated in the design of the study and high-throughput sequencing data acquisition. KJC and EYC provided intellectual criticisms and gave final approval to the manuscript to be published. All authors read and approved the final manuscript.

\section{Authors' information}

WHK and CSH are breast cancer surgeons at National Taiwan University Hospital. LCL is a professor of Physiology at National Taiwan University. MHT is a professor of Biotechnology at National Taiwan University. KJC is a professor of Breast Surgery at National Taiwan University. EYC is a professor of Biomedical Electronics and Bioinformatics at National Taiwan University.

\section{Acknowledgements}

This study was supported by grants from the Department of Health Taiwan (DOH101-TDC-111-001) and the National Science Council, Taiwan (NSC97-2314-B-002-154-MY3). The sequencing data analyses were supported by the Center of Biotechnology at National Taiwan University. We thank Genelabs Life Science (Taiwan) for performing STR-profiling authentication of the cell lines used in this study.

\section{Author details}

'Department of Electrical Engineering, Graduate Institute of Biomedical Electronics and Bioinformatics, National Taiwan University, Taipei, Taiwan. ${ }^{2}$ Department of Surgery, College of Medicine, National Taiwan University, Taipei, Taiwan. ${ }^{3}$ Bioinformatics and Biostatistics Core, NTU Center of Genomic Medicine, Taipei, Taiwan. ${ }^{4}$ Institute of Biotechnology, College of Bio-resources and Agriculture, National Taiwan University, Taipei, Taiwan. ${ }^{5}$ Department of Obstetrics and Gynecology, College of Medicine, National Taiwan University, Taipei, Taiwan. ${ }^{6}$ Department of Physiology, College of Medicine, National Taiwan University, Taipei, Taiwan. 'Department of Surgery, Cheng Ching General Hospital, Taichung, Taiwan. 


\section{References}

1. Foulkes WD, Smith IE, Reis-Filho JS. Triple-negative breast cancer. N Engl J Med. 2010;363:1938-48.

2. Cleator S, Heller W, Coombes RC. Triple-negative breast cancer: therapeutic options. Lancet Oncol. 2007;8:235-44.

3. Van't Veer $L$, Dai H, van de Vijver MJ, He YD, Hart AA, Mao M, et al. Gene expression profiling predicts clinical outcome of breast cancer. Nature. 2002;415:530-6.

4. Kreike $B$, van Kouwenhove $M$, Horlings $H$, Weigelt $B$, Peterse $H$, Bartelink $H$, et al. Gene expression profiling and histopathological characterization of triple-negative/basal-like breast carcinomas. Breast Cancer Res: BCR. 2007;9:R65.

5. Krol J, Loedige I, Filipowicz W. The widespread regulation of microRNA biogenesis, function and decay. Nat Rev Genet. 2010;11:597-610.

6. Winter J, Jung S, Keller S, Gregory Rl, Diederichs S. Many roads to maturity: microRNA biogenesis pathways and their regulation. Nat Cell Biol. 2009;11:228-34.

7. Volinia S, Calin GA, Liu CG, Ambs S, Cimmino A, Petrocca F, et al. A microRNA expression signature of human solid tumors defines cancer gene targets. Proc Natl Acad Sci U S A. 2006;103:2257-61.

8. Iorio MV, Ferracin M, Liu CG, Veronese A, Spizzo R, Sabbioni S, et al. MicroRNA gene expression deregulation in human breast cancer. Cancer Res. 2005:65:7065-70.

9. Hayashita Y, Osada H, Tatematsu Y, Yamada H, Yanagisawa K, Tomida S, et al. A polycistronic microRNA cluster, miR-17-92, is overexpressed in human lung cancers and enhances cell proliferation. Cancer Res. 2005;65:9628-32.

10. Si ML, Zhu S, Wu H, Lu Z, Wu F, Mo YY. miR-21-mediated tumor growth. Oncogene. 2007;26:2799-803.

11. Sachdeva M, Zhu S, Wu F, Wu H, Walia V, Kumar S, et al. p53 represses c-Myc through induction of the tumor suppressor miR-145. Proc Natl Acad Sci U S A. 2009:106:3207-12.

12. Calin GA, Croce CM. MicroRNA-cancer connection: the beginning of a new tale. Cancer Res. 2006:66:7390-4.

13. Schmittgen TD, Livak KJ. Analyzing real-time PCR data by the comparative $C$ (T) method. Nat Protoc. 2008:3:1101-8.

14. Horne MC, Donaldson KL, Goolsby GL, Tran D, Mulheisen M, Hell JW, et al. Cyclin $\mathrm{G} 2$ is up-regulated during growth inhibition and B cell antigen receptor-mediated cell cycle arrest. J Biol Chem. 1997;272:12650-61.

15. Bennin DA, Don AS, Brake T, McKenzie JL, Rosenbaum H, Ortiz L, et al. Cyclin $\mathrm{G} 2$ associates with protein phosphatase $2 \mathrm{~A}$ catalytic and regulatory $\mathrm{B}^{\prime}$ subunits in active complexes and induces nuclear aberrations and a G1/S phase cell cycle arrest. J Biol Chem. 2002;277:27449-67.

16. Ito $Y$, Yoshida H, Uruno T, Nakano K, Takamura Y, Miya A, et al. Decreased expression of cyclin $\mathrm{G} 2$ is significantly linked to the malignant transformation of papillary carcinoma of the thyroid. Anticancer Res. 2003:23:2335-8

17. Kim Y, Shintani S, Kohno Y, Zhang R, Wong DT. Cyclin G2 dysregulation in human oral cancer. Cancer Res. 2004;64:8980-6.

18. Radojicic J, Zaravinos A, Vrekoussis T, Kafousi M, Spandidos DA, Stathopoulos EN. MicroRNA expression analysis in triple-negative (ER, PR and Her2/neu) breast cancer. Cell Cycle. 2011;10:507-17.

19. Cascione L, Gasparini P, Lovat F, Carasi S, Pulvirenti A, Ferro A, et al. Integrated microRNA and mRNA signatures associated with survival in triple negative breast cancer. PLoS One. 2013;8:e55910.

20. Kitago M, Martinez SR, Nakamura T, Sim MS, Hoon DS. Regulation of RUNX3 tumor suppressor gene expression in cutaneous melanoma. Clinical Cancer Res. 2009;15:2988-94.

21. Yanaihara N, Caplen N, Bowman E, Seike M, Kumamoto K, Yi M, et al. Unique microRNA molecular profiles in lung cancer diagnosis and prognosis. Cancer Cell. 2006;9:189-98.

22. Slaby O, Svoboda M, Fabian P, Smerdova T, Knoflickova D, Bednarikova M, et al. Altered expression of miR-21, miR-31, miR-143 and miR-145 is related to clinicopathologic features of colorectal cancer. Oncology. 2007;72:397-402

23. Ostenfeld MS, Bramsen JB, Lamy P, Villadsen SB, Fristrup N, Sorensen KD, et al. miR-145 induces caspase-dependent and -independent cell death in urothelial cancer cell lines with targeting of an expression signature present in Ta bladder tumors. Oncogene. 2010;29:1073-84.
24. Shi B, Sepp-Lorenzino L, Prisco M, Linsley P, de Angelis T, Baserga R. Micro RNA 145 targets the insulin receptor substrate-1 and inhibits the growth of colon cancer cells. J Biol Chem. 2007;282:32582-90.

25. Sachdeva M, Mo YY. MicroRNA-145 suppresses cell invasion and metastasis by directly targeting mucin 1. Cancer Res. 2010;70:378-87

26. Li D, Zhao Y, Liu C, Chen X, Qi Y, Jiang Y, et al. Analysis of MiR-195 and MiR-497 expression, regulation and role in breast cancer. Clinical Cancer Res. 2011;17:1722-30

27. Xu T, Zhu Y, Xiong Y, Ge YY, Yun JP, Zhuang SM. MicroRNA-195 suppresses tumorigenicity and regulates G1/S transition of human hepatocellular carcinoma cells. Hepatology. 2009;50:113-21.

28. Alevizos I, Mahadevappa M, Zhang X, Ohyama H, Kohno Y, Posner M, et al. Oral cancer in vivo gene expression profiling assisted by laser capture microdissection and microarray analysis. Oncogene. 2001;20:6196-204.

29. Frasor J, Danes JM, Komm B, Chang KC, Lyttle CR, Katzenellenbogen BS. Profiling of estrogen up- and down-regulated gene expression in human breast cancer cells: insights into gene networks and pathways underlying estrogenic control of proliferation and cell phenotype. Endocrinology. 2003;144:4562-74

30. Ahmed S, Al-Saigh S, Matthews J. FOXA1 is essential for aryl hydrocarbon receptor-dependent regulation of cyclin G2. Molecular Cancer Res. 2012;10:636-48

31. Stossi F, Likhite VS, Katzenellenbogen JA, Katzenellenbogen BS. Estrogen-occupied estrogen receptor represses cyclin G2 gene expression and recruits a repressor complex at the cyclin G2 promoter. J Biol Chem. 2006:281:16272-8.

32. Fu G, Peng C. Nodal enhances the activity of FoxO3a and its synergistic interaction with Smads to regulate cyclin G2 transcription in ovarian cancer cells. Oncogene. 2011;30:3953-66.

33. Montagner M, Enzo E, Forcato M, Zanconato F, Parenti A, Rampazzo E, et al. SHARP1 suppresses breast cancer metastasis by promoting degradation of hypoxia-inducible factors. Nature. 2012;487:380-4.

34. Schulte $J H$, Marschall T, Martin M, Rosenstiel P, Mestdagh P, Schlierf S, et al. Deep sequencing reveals differential expression of microRNAs in favorable versus unfavorable neuroblastoma. Nucleic Acids Res. 2010;38:5919-28.

35. Betel D, Wilson M, Gabow A, Marks DS, Sander C. The microRNA.org resource: targets and expression. Nucleic Acids Res. 2008;36:D149-53.

36. Paraskevopoulou MD, Georgakilas G, Kostoulas N, Vlachos IS, Vergoulis T, Reczko $M$, et al. DIANA-microT web server v5.0: service integration into miRNA functional analysis workflows. Nucleic Acids Res. 2013;41:W169-73.

37. Vergoulis T, Vlachos IS, Alexiou P, Georgakilas G, Maragkakis M, Reczko M, et al. TarBase 6.0: capturing the exponential growth of miRNA targets with experimental support. Nucleic Acids Res. 2012;40:D222-9.

38. Xiao F, Zuo Z, Cai G, Kang S, Gao X, Li T. miRecords: an integrated resource for microRNA-target interactions. Nucleic Acids Res. 2009;37:D105-10.

39. Lu TP, Lee CY, Tsai MH, Chiu YC, Hsiao CK, Lai LC, et al. miRSystem: an integrated system for characterizing enriched functions and pathways of microRNA targets. PLoS One. 2012;7:e42390.

40. Weiner MP, Costa GL, Schoettlin W, Cline J, Mathur E, Bauer JC. Site-directed mutagenesis of double-stranded DNA by the polymerase chain reaction. Gene. 1994;151:119-23.

\section{Submit your next manuscript to BioMed Central and take full advantage of:}

- Convenient online submission

- Thorough peer review

- No space constraints or color figure charges

- Immediate publication on acceptance

- Inclusion in PubMed, CAS, Scopus and Google Scholar

- Research which is freely available for redistribution 